\title{
Aplicación del derecho y la justificación de las decisiones judiciales
}

\author{
Law-application and the Justification of \\ Judicial Decisions
}

Luís Duarte d'Almeida ${ }^{* *}$

\begin{abstract}
Resumen: Este artículo responde a los comentarios y críticas presentados por Hugo Zuleta, María Gabriela Scataglini y José Juan Moreso no solo a “¿Qué es aplicar derecho?”, sino también a un artículo anterior, “On the Legal Syllogism” (“Sobre el silogismo jurídico”). Se discute el deductivismo de reglas y el modelo del silogismo jurídico, y se aclara, defiende y desarrolla el modelo de la aplicabilidad de la justificación de las decisiones judiciales propuesto en “¿Qué es aplicar derecho?”.

Palabras clave: silogismo jurídico, deductivismo de reglas, justificación de decisiones judiciales, particularismo, enunciados de segundo orden, aplicabilidad.
\end{abstract}

\begin{abstract}
This article replies to the comments and criticisms made by Hugo Zuleta, María Gabriela Scataglini and José Juan Moreso not only to "What Is It to Apply the Law?", but to a previous related article, "On the Legal Syllogism". It discusses rule-deductivism and the model of the legal syllogism, and clarifies, defends, and develops the applicability model of
\end{abstract}

Traducción de Sebastián Figueroa. Revisada por el autor.

** Professor of Jurisprudence, Edinburgh Law School, University of Edinburgh, Reino Unido. Correo electrónico: luis.duarte.almeida@ed.ac.uk 
the justification of law-applying judicial decisions proposed in "What Is It to Apply the Law?"

Keywords: legal syllogism, rule-deductivism, law-application, justification of judicial decisions, particularism, second-order claims, applicability.

\section{Introducción}

Quisiera comenzar con una nota personal. Conozco a Gabriela Scataglini, Hugo Zuleta y José Juan Moreso desde hace bastantes años y, de hecho, cuando pienso en los inicios de mi carrera en la filosofía del derecho, pienso también en ellos.

La primera ponencia propiamente dicha que presenté, en el primer congreso internacional al que asistí, fue en el XXII IVR World Congress on Philosophy of Law and Social Philosophy, en Granada, en mayo de 2005. Era yo uno de los dos únicos ponentes portugueses (el otro era mi querido profesor José de Sousa e Brito, a quien debo el descubrimiento de la filosofía del derecho). Al no conocer a casi nadie, me sentía un poco perdido entre esos cientos de personas. El primer día, durante el almuerzo, llevaba mi bandeja de comida en busca de una silla libre, y encontré una en una mesa que, por lo demás, ya estaba llena. Y descubrí, para mi placer, que en esa mesa había una serie de personas cuyos nombres reconocía por haber leído y aprendido de trabajos que habían publicado.

Entre el grupo estaba Gabriela; también era su primera conferencia internacional. Gabriela fue, por tanto - junto con Juan Pablo Alonso, del que guardo recuerdos igualmente gratos - una de las primeras colegas internacionales que conocí personalmente y con los que me hice amigo. Gabriela y Juan Pablo tuvieron la amabilidad de asistir a mi ponencia unos días después (yo estaba todavía muy verde, y me encontraba muy nervioso) y aún recuerdo sus útiles y generosos comentarios (así como la noche que siguió: también era mi cumpleaños).

Luego, gracias a ese encuentro casual, me invitaron a visitar Buenos Aires en 2006 para presentar otra ponencia en el famoso seminario que Carlos Alchourrón y Eugenio Bulygin habían iniciado décadas atrás. Y fue entonces cuando conocí a Hugo (y a Eugenio). Aquel fue el primero 
de varios viajes a Argentina, y he tenido la suerte de mantener muchas discusiones estimulantes ( $\mathrm{y}$ de disfrutar de varias cenas excelentes) tanto con Gabriela como con Hugo (y con Juan Pablo) a lo largo de los años.

En cuanto a ese primer trabajo mío, el editor del volumen de ARSP: Beihefte en el que se publicó fue José Juan. Y desde entonces, también he estado con él en muchos contextos (y países; y excelentes cenas también), y siempre me he beneficiado mucho de sus comentarios, consejos y contagiosa afabilidad.

Así que verme ahora, más de 15 años después de aquellos primeros encuentros, en la envidiable posición de ser el destinatario de los incisivos y provocadores comentarios de Gabriela, Hugo y José Juan es para mí una fuente no solo de satisfacción profesional sino también de alegría personal. Les agradezco mucho que se hayan tomado la tarea de discutir mi trabajo. Y espero que encuentren que mis respuestas han hecho justicia a sus comentarios. Ciertamente, me han hecho trabajar bastante.

Estoy muy agradecido también a Sebastián Figueroa (un amigo más reciente, pero un compañero igualmente apreciado tanto de debates filosóficos como de cenas no filosóficas; a los filósofos del derecho, al menos a los de países latinos, nos gusta cenar bien) por promover este intercambio sobre mi trabajo y por traducir cuidadosamente mis artículos; y a Federico Arena, por acogerlo en Discusiones.

Y ahora, discutamos.

\section{Respuesta a Hugo Zuleta}

Zuleta dedica la mayor parte de su comentario a discutir, no el artículo que aparece en el presente número de Discusiones, sino un artículo mío anterior, "On the Legal Syllogism" (Duarte d'Almeida, 2019). En ese trabajo, como señala Zuleta, defiendo puntos de vista que “¿Qué es aplicar derecho?” (Duarte d'Almeida, 2021) presupone o desarrolla. Y, en efecto, los dos artículos pueden verse como piezas complementarias. (Como dice José Juan Moreso en su comentario, el primero aporta la pars destruens, el segundo la pars construens). Por lo tanto, me complace tener la oportunidad de discutir también ese artículo anterior. Así que empezaré por 
abordar lo que dice Zuleta al respecto, antes de pasar a sus observaciones sobre “¿Qué es aplicar derecho?”.

Sin embargo, debo comenzar dando a los lectores de Discusiones una rápida visión general de algunas de las ideas que defiendo en "On the Legal Syllogism". Esto importa en parte porque esa obra no está incluida en este volumen de Discusiones, y porque fue publicada solo en inglés. Pero importa también, y principalmente, porque hay puntos importantes que hago explícitamente en "On the Legal Syllogism" que tienen que ver directamente con las observaciones críticas que hace Zuleta, pero que este no menciona.

\subsection{Tres características del deductivismo de reglas}

Mi objetivo en "On the Legal Syllogism” era poner al descubierto las deficiencias de una concepción muy popular sobre la justificación de las decisiones judiciales que aplican derecho. Según esa concepción, cuando un tribunal aplica el derecho, la justificación de su decisión puede reconstruirse como un argumento deductivo cuyas premisas son (a) un enunciado de una regla jurídica y (b) un enunciado de los hechos relevantes de un caso concreto; y cuya conclusión afirma la respectiva consecuencia en ese caso concreto. Esto es lo que comúnmente se denomina "silogismo jurídico" (o "silogismo judicial”). Y la opinión de que la justificación de las decisiones judiciales que aplican derecho se reconstruye adecuadamente como un silogismo jurídico es lo que llamo "deductivismo de reglas". El deductivismo de reglas es ampliamente adoptado en la teoría jurídica contemporánea.

En ese artículo sostengo que el deductivismo de reglas es una concepción errónea, pero no sin antes explicar cuidadosamente cómo debe entenderse exactamente esa concepción - ya que incluso sus defensores no suelen tenerlo del todo claro- $-{ }^{1} \mathrm{Y}$ entre las diversas características del deductivismo de reglas que me ocupo de destacar y explicar, hay tres que me gustaría mencionar ahora.

1 La parte II de mi artículo, en la que presento mi caracterización del deductivismo de reglas, ocupa casi un tercio de todo el texto: véase Duarte d'Almeida (2019, pp. 337-345). 
En primer lugar, el deductivismo de reglas se ocupa de la justificación argumentativa de las decisiones judiciales que aplican derecho, no del proceso mental de razonar por el que se llega a esas decisiones. El punto se plantea a menudo - como digo explícitamente en mi artículo (Duarte d'Almeida, 2019, p. 341) - en términos de la distinción (importada de la filosofía de la ciencia) entre justificación y descubrimiento. El deductivismo de reglas no tiene nada que decir sobre el "proceso habitual o los hábitos de pensamiento por los que los jueces realmente llegan a sus decisiones", como escribe H. L. A. Hart (que también era un deductivista de reglas). Esas son cuestiones de "psicología descriptiva"; el deductivismo de reglas se refiere en cambio a "la evaluación o justificación de las decisiones".

En segundo lugar, el deductivismo de reglas es, más precisamente, un planteamiento sobre el contenido y la estructura lógica de los argumentos que los tribunales suelen dar cuando justifican sus decisiones que aplican el derecho. Esto tiene tres implicaciones importantes. La primera es que los deductivistas de reglas no afirman que los jueces, en las justificaciones escritas que normalmente dan de las decisiones que aplican derecho, de hecho, ofrecen silogismos jurídicos. Lo que los deductivistas de reglas afirman, como también explico en mi artículo, es que los argumentos que los jueces de hecho ofrecen pueden reconstruirse como instancias de silogismos jurídicos (Duarte d'Almeida, 2019, pp. 339-340).

Pero - y esta es la segunda implicación — hay criterios para lo que puede contar como buena reconstrucción de un argumento, y uno de esos criterios (que impone una restricción de "ajuste", de " $f i t$ ") es que la reconstrucción capte el argumento que realmente fue dado por la persona que lo presentó. Y la tercera implicación es que cualquiera que ofrezca una reconstrucción de un argumento tiene que sostener que la persona que lo presentó está comprometida, aunque solo implícitamente, con la verdad de las premisas (y de la conclusión) del argumento tal y como aparecen en la reconstrucción.

En otras palabras, quien ofrece una reconstrucción de un argumento tiene que sostener que las premisas, tal y como aparecen en la reconstrucción, son afirmaciones que es plausible atribuir a quien presentó el

2 Véase: Hart (1967, p. 105). Respecto del compromiso de Hart con el deductivismo de reglas, véase también, por ejemplo, Hart (1955, pp. 239-264) y Hart (2012, pp. 126-127). 
argumento como afirmaciones que esa persona sostiene y en las que basa argumentativamente su conclusión. (Esto no supone que quien presentó el argumento haya formulado explícitamente tales afirmaciones en la forma que aparecen en la reconstrucción; muchos argumentos, si no la mayoría, se ofrecen informalmente en forma entimemática). Los deductivistas de reglas sostienen, por lo tanto, que las premisas y la conclusión en el silogismo jurídico son afirmaciones que los jueces que aplican derecho de hecho sostienen, o con las que están comprometidos.

Y el tercer rasgo importante del deductivismo de reglas es que el silogismo jurídico no pretende ser una reconstrucción completa de la estructura argumentativa de las decisiones judiciales que aplican derecho (Duarte d'Almeida, 2019, pp. 341-342). De hecho, los deductivistas de reglas suelen señalar que cada una de las premisas del silogismo jurídico necesita (por supuesto) una justificación adicional, y que los jueces con frecuencia (e incluso típicamente) dan argumentos para apoyarlas. El silogismo jurídico, sostienen los deductivistas de reglas, reconstruye solo el último eslabón lógico de la cadena argumentativa (Duarte d'Almeida, 2019, p. 342).

Mi principal razón para mencionar estas tres características es que me parece que, en general, están ausentes de la forma en que el propio Zuleta presenta y concibe el deductivismo de reglas $-\mathrm{y}$ por lo tanto ausentes también de lo que él sugiere a sus lectores que yo considero que es el deductivismo de reglas-. Esta ausencia subyace a, y socava, como veremos, algunos de sus comentarios críticos sobre mis puntos de vista.

Zuleta dice que las "principales objeciones" que presento contra el deductivismo de reglas son que (a) "no reconstruye adecuadamente la práctica judicial” y que $(b)$ "el silogismo jurídico no expresa que la regla formulada en las premisas se aplica al caso a resolver" (Zuleta, 2021, sección 3). Esto último lo considera, en efecto, "el principal problema" que encuentro en el silogismo jurídico: "que en ningún lugar [del silogismo jurídico] se dice que la disposición legal se aplica al caso en consideración" (Zuleta, 2021, sección 1). Esto, como veremos (en la sección 2.7), no es completamente correcto. Pero Zuleta dedica la mayor parte de su tiempo a intentar refutar la primera objeción.

Sus intentos de refutación, sin embargo, fracasan. También veremos por qué. 


\subsection{El deductivismo de reglas y la práctica judicial}

Como señala Zuleta, yo creo que el deductivismo de reglas atribuye injustificadamente a los jueces un compromiso con afirmaciones del tipo de las que figuran como la premisa jurídica general del silogismo jurídico.

¿Por qué digo que el deductivismo de reglas atribuye tal compromiso a los jueces? Porque, como he explicado en la sección anterior, el silogismo jurídico pretende proporcionar una reconstrucción satisfactoria de los argumentos que los tribunales dan para justificar decisiones que aplican derecho. Ahora bien, el silogismo jurídico incluye, como premisa, el enunciado de una regla jurídica universal. Y ninguna reconstrucción de un argumento tiene éxito si los elementos que lo componen — sus premisas y su conclusión - son afirmaciones que no es plausible considerar que la persona que ofrece el argumento toma como verdaderas. Por lo tanto, el deductivismo de reglas debe sostener que los jueces sí toman tales enunciados de reglas universales como verdaderos.

Pero esta atribución, sostengo, simplemente no es plausible. ¿Por qué no? Hay varias razones.

La primera es que los jueces, típicamente, de hecho no se comprometen con tales enunciados como premisas en sus argumentos. Esto lo confirma la propia práctica judicial. Y, en efecto, ¿por qué habrían de comprometerse con tales enunciados universales? Para sostener como verdadera una afirmación de ese tipo, uno necesita estar seguro de que la regla enunciada vale pase lo que pase. Es decir, uno tiene que estar seguro de que la consecuencia especificada vale para cualquier caso que satisfaga el antecedente de la regla: que la formulación de la regla no es sobre-incluyente. Pero los jueces no necesitan sostener eso para cumplir con su tarea - la tarea de decidir el caso concreto en cuestión-.

Lo que los jueces necesitan es sostener que el caso concreto en cuestión es tal que la disposición relevante se aplica a él. Esto, claro, significa —como señalo en "On the Legal Syllogism" - que los jueces están racionalmente comprometidos con la idea de que la disposición se aplica también a cualquier otro caso que sea similar en todos los aspectos relevantes al caso en cuestión. Las decisiones judiciales, en otras palabras, se asientan sobre bases universalizables (Duarte d'Almeida 2019, p. 357). Pero esto no quiere 
decir que los jueces tengan que formarse (y mucho menos presentar) una opinión sobre cuales son exactamente los aspectos relevantes del caso en cuestión -que es lo que tendrían que hacer para comprometerse con formulaciones de reglas universales-.

Y no se trata simplemente de que los jueces no formulen a menudo tales enunciados. Cuando de hecho se aventuran a dar formulaciones de reglas, como ocurre a veces, suelen dejar claro que consideran sus formulaciones como meras aproximaciones tentativas de la regla pertinente. Y para más evidencia de que tales formulaciones no se presentan como enunciados verdaderos de reglas universales, podemos observar cómo reaccionan a ellas otros tribunales. Esto es así incluso en jurisdicciones en las que los tribunales están obligados a seguir los precedentes: no es la función de un juez "presentar definiciones o formular reglas de manera rígida", dice Lord Reid en Cassel v Broome; y cuando se aventuran a ofrecer formulaciones de reglas, "mucho de lo que dicen pretende ser ilustrativo o explicativo y no definitivo". ${ }^{3}$

Y esto, después de todo, es de esperar. Así suele proceder nuestro razonamiento práctico: no desde principios precisamente articulados hacia juicios normativos particulares, sino desde juicios particulares hacia articulaciones tentativas de los principios que los justificarían. Además (como ilustra el trabajo filosófico contemporáneo sobre ética práctica), el proceso de intentar articular formulaciones rigurosas de los principios que justifican nuestros juicios normativos particulares es un proceso difícil. Requiere que constantemente pongamos a prueba y revisemos, frente a una serie de contraejemplos, muchas formulaciones posibles de reglas o principios. $\mathrm{Y}$ eso, de nuevo, es algo que los jueces ni siquiera intentan hacer.

Como dice Simpson, el hecho de que un juez deba, en cierto sentido, tener "en mente" una regla cuando "decide actuar" no significa que "deba tener en mente una formulación precisa de una regla; una persona puede

3 Cassell \& Co Ltd v Broome [1972] 2 WLR 645 at 681-2. Véase también Simpson, 1961. Este indica: "Incluso cuando un juez pone un cuidado especial en formular una regla con exactitud y precisión, no solemos tratar dicha formulación del mismo modo que un artículo de una ley, ya que la prerrogativa de los jueces no es conferir fuerza vinculante a una regla formulándola [...] sino más bien decidir los casos actuando en base a las reglas, sin fijar para el futuro la forma verbal de la regla sobre la base de una única aplicación de la misma" (Simpson, 1961, p. 162). 
actuar en base a una regla sin pensar en cómo redactarla" (Simpson, 1961, p. 162). Y la cuestión, nótese, no es simplemente una cuestión de razonamiento. Se traslada al ámbito de la justificación. Y eso es porque nuestros juicios normativos particulares no son ni irracionales ni incapaces de ser defendidos argumentativamente en ausencia de tales formulaciones de reglas precisas. La cuestión, por tanto, es también que es posible argumentar a favor de tales juicios particulares - justificarlos satisfactoriamentesin tener que explicitar y tomar por verdaderos enunciados precisos de reglas universales. ${ }^{4}$

Digo todo esto para aclarar mi tesis de que el deductivismo de reglas y el modelo del silogismo jurídico no corresponden a la práctica real de los tribunales. Todos estos puntos ya los había expuesto en "On the Legal Syllogism"; pero necesitaba hacerlo aquí también, porque Zuleta no se ocupa de ellos. Zuleta interpreta mi punto de vista de una manera muy diferente - lo malinterpreta, en realidad- y me atribuye (y luego trata de refutar) un argumento que yo no he presentado, ni adoptaría.

Empezaré a discutir sus objeciones en la sección 2.4. Antes de hacerlo, sin embargo, todavía es necesaria una breve nota sobre la distinción que propuse en “¿Qué es aplicar derecho?”- entre aplicación inferencial y aplicación pragmática del derecho. Es una distinción con la que Zuleta está de acuerdo (Zuleta, 2021, sección 6). Pero dado que Zuleta se basa en ella (erróneamente, creo) para criticar mi propia afirmación de que el deductivismo de reglas no da cuenta de la práctica judicial, es importante que aclare los aspectos cruciales de la distinción. Y también será útil a las lectoras y a los lectores de estas respuestas tener ahora visibles ante sí los esquemas que propuse.

\subsection{Aplicación inferencial y aplicación pragmática del derecho}

Así es como propongo definir a la aplicación inferencial del derecho:

4 Regresaré a este tema, en la sección 4.2, al responder a los comentarios de José Juan Moreso. 
Aplicar inferencialmente una disposición $p$ a un determinado objeto $x$ es razonar hacia una conclusión $c$ respecto de $x$ sobre la base de que (a) $p$ se aplica a $x$, y (b) se aplica a $x$, entonces $c$ es jurídicamente verdadera.

Mientras que mi definición de la aplicación pragmática del derecho es la siguiente:

Aplicar pragmáticamente una disposición $p$ es realizar una acción $\varphi$ tal que:

(a) el agente considera que $\varphi$ es una acción que o bien (a1) debe (todo considerado) jurídicamente realizar, o bien al menos (a2) le está jurídicamente permitido realizar (en el sentido de que no es el caso de que, todo considerado, no deba jurídicamente realizarla);

(b) el agente considera que el juicio de deber relevante — es decir, (a1) o (a2) - se apoya en la conclusión de la aplicación inferencial de $p$ a un determinado objeto $x ; \mathrm{y}$

(c) realizando $\varphi$, el agente pretende estar resolviendo autoritativamente una determinada cuestión o controversia.

Y abajo doy lo que considero el esquema de la estructura de la justificación de las decisiones judiciales que aplican derecho. No es un esquema que ofrezca explícitamente en mi artículo, pero corresponde a la reconstrucción que allí presenté del argumento de Lord Denning en The Hollandia (y Zuleta ofrece un esquema idéntico al final de la sección 2 de su comentario):

(1) Si la disposición $p$ se aplica a $x$, entonces $c$.

(2) La disposición $p$ se aplica a $x$.

Por lo tanto (de (1) y (2)),

(3) $c$.

(4) Si $c$, y no hay consideraciones compensatorias [i.e. consideraciones de igual o mayor fuerza en sentido contrario], entonces, todo considerado, este tribunal debe jurídicamente (o no es el caso que no deba jurídicamente) $\varphi$. 
(5) No hay consideraciones compensatorias.

Por lo tanto (de (3), (4) y (5))

(6) Todo considerado, este tribunal debe jurídicamente (o no es el caso que no deba jurídicamente) $\varphi$.

Es la estructura de un argumento complejo: una cadena de dos argumentos, el primero (de (1) y (2) hacia (3)) correspondiente a la aplicación inferencial del derecho, el segundo (de (3), (4) y (5) hacia (6)) a la aplicación pragmática del derecho. Utilizo " $p$ " para representar la disposición que se aplica, " $x$ " para el objeto directo de la aplicación (es decir, aquello a lo que se aplica la disposición), “c" para el enunciado que el tribunal considera verdadero como cuestión de derecho, y “ $\varphi$ ” para la decisión relevante.

\subsection{Zuleta sobre reglas universales y el compromiso de los jueces hacia ellas}

Zuleta está de acuerdo conmigo, como ya he mencionado, en que debemos distinguir entre aplicación inferencial y aplicación pragmática del derecho (Zuleta, 2021, sección 6). Pero Zuleta piensa que la distinción puede a su vez servir para responder a mi afirmación de que no tenemos motivos para atribuir a los jueces un compromiso con enunciados de reglas universales como premisas de sus argumentos.

Así es como Zuleta ve las cosas. Hay una diferencia, dice, entre la identificación de las reglas jurídicas relevantes - lo que implica identificar las condiciones que aparecen en sus antecedentes- y la identificación de las condiciones de las que depende su aplicación. La identificación de una regla jurídica tiene en cuenta cualquier excepción "intrasistémica" relevante (es decir, cualquier "circunstancia derrotante" que esté "contemplada en otras normas del mismo sistema jurídico") (Zuleta, 2021, sección 3.1). Dado que estas excepciones pueden ser, "en teoría" al menos, "identificadas exhaustivamente", la "ausencia de tales circunstancias" puede incorporarse "al antecedente de la regla" (Zuleta, 2021, sección 3.1).

Una regla cuyo antecedente es satisfecho por los hechos de un caso es, para Zuleta, aplicable a ese caso. Pero el hecho de que una regla sea aplica- 
ble a un caso le da al tribunal, dice Zuleta, solo "una razón para decidir [el caso] de acuerdo con lo que la disposición establece". Y esta razón puede bien ser derrotada, porque las reglas jurídicas pueden también estar sujetas a excepciones "extra-sistémicas" (es decir, a "circunstancias derrotantes" que provengan de "normas de otros sistemas, como la moral, por ejemplo") (Zuleta, 2021, sección 3.1). Tales excepciones extra-sistémicas pueden surgir, y ser relevantes, en el momento de la aplicación, y proporcionar "consideraciones compensatorias" de mayor peso que, todo considerado, justificarán la no aplicación de la regla jurídica aplicable.

Así, aunque las excepciones extra-sistémicas no tienen que ver con la "cuestión teórica" de cómo identificar la regla jurídica relevante, sí tienen que ver con la "cuestión práctica" de si una regla aplicable debe ser aplicada. Y Zuleta considera que este último punto también lo hago yo, o al menos que puedo estar de acuerdo con él, dado que en mi propia propuesta señalo - como pone de manifiesto el esquema que presenté al final de la sección 2.3- que la aplicación pragmática de una disposición depende de la ausencia de consideraciones compensatorias.

"Con esto en mente", sin embargo, dice Zuleta, se puede demostrar que es falsa mi tesis de que el planteamiento de los deductivistas de reglas de que los tribunales se basan en reglas universales como premisas en sus argumentos implica atribuir a los tribunales "el compromiso de resolver de la misma manera siempre que se cumplan los supuestos descritos en el antecedente de la regla",

ya que la decisión que en definitiva se adopta en cada circunstancia pertenece al ámbito de la aplicación pragmática, como el mismo Duarte d'Almeida advierte acertadamente, no al de la determinación de la solución que prescribe la norma, que correspondería a la etapa inferencial (Zuleta, 2021, sección 3.1).

“En otras palabras", escribe,

el hecho de que pueda reconstruirse racionalmente la decisión de un juez como derivada de una regla general nada dice sobre la regla que va a aplicar ese mismo juez la siguiente vez que tenga que 
Aplicación del derecho y la justificación de las decisiones judiciales

decidir en un caso que reúna las características contempladas en el antecedente de la regla (Zuleta, 2021, sección 3.1).

Pero esto no es ni riguroso ni correcto.

En primer lugar, un punto menor, pero importante. No hay conexión directa entre la noción de "consideraciones compensatorias" tal y como la utilizo, y el tema de la derrotabilidad $-\mathrm{y}$ mucho menos con la noción de derrotabilidad "extra-sistémica" (que se refiere, como hemos visto, a "circunstancias derrotantes" extra-jurídicas que "provienen" de "normas de otros sistemas, como la moral, por ejemplo") (Zuleta, 2021, sección 3.1)-. Una consideración compensatoria - es decir, una consideración de igual o mayor fuerza en sentido contrario- no es lo mismo que una excepción o circunstancia derrotante. Además, tampoco creo - y no lo sugiero en mi artículo- que las consideraciones compensatorias que puedan afectar al resultado de la aplicación pragmática del derecho deban ser consideraciones no jurídicas, o "extra-jurídicas". Zuleta dice a sus lectores que la noción de derrotabilidad "aparece” (Zuleta, 2021, sección 3.1) en mi propuesta tanto en el nivel de la aplicación inferencial del derecho como en el de la aplicación pragmática del derecho, pero no es así: la noción de derrotabilidad está completamente ausente de cualquiera de mis artículos, y no me amparo en ella. ${ }^{5}$

Pero, en segundo lugar, un punto más importante. Mi postura no es que si consideramos - como los deductivistas de reglas- que los jueces están comprometidos con enunciados de reglas universales, les estemos atribuyendo "el compromiso de resolver de la misma manera siempre que se cumplan los supuestos descritos en el antecedente de la regla." (Zuleta, 2021, sección 3.1). Lo que afirmo no se refiere a lo que los jueces se comprometen a decidir en la fase pragmática de aplicación del derecho. Esto lo digo claramente también. En la sección III de "On the Legal Syllogism", de hecho, explico por qué no es plausible interpretar al silogismo jurídico como modelo de justificación de decisiones (Duarte d'Almeida, 2019, pp. 346-350). Más bien, como explico en la sección IV de ese artículo, el silo-

5 He discutido ampliamente la noción de derrotabilidad en mi libro Allowing for Exceptions: A Theory of Defences and Defeasibility in Law (Duarte d'Almeida, 2015a). 
gismo jurídico es un modelo de justificación de las proposiciones jurídicas particulares que los jueces toman como verdaderas sobre la base de que una regla (como dirían los deductivistas de reglas) se aplica a un objeto en particular (Duarte d'Almeida, 2019, pp. 350-359). En otras palabras, lo que el silogismo jurídico intenta (pero no logra) modelar es lo que yo llamo aplicación inferencial del derecho. Y explico también que la conclusión relevante - la proposición jurídica particular relevante- no se correlaciona invariablemente con la misma decisión en cualquier caso posible.

Por lo tanto, mi punto sobre universalizabilidad no es en absoluto un punto sobre la aplicación pragmática del derecho. Es un punto sobre la aplicación inferencial del derecho. Y el punto es simplemente que, si tomamos a los jueces como comprometidos con tales enunciados de reglas universales, les estamos atribuyendo un compromiso con enunciados que (como expliqué en la sección 2.2) no es plausible considerar que tomen como verdaderos.

Al final de su artículo, Zuleta vuelve a lo que él llama mi "rechazo de reglas universales" para hacer la siguiente observación:

[M] e parece que es necesario aclarar cuál es el propósito de la teoría del silogismo jurídico. Si lo que se pretendiera fuera reconstruir el proceso mental por el que el juez arriba a la decisión del caso, tendría razón Duarte d'Almeida. Pero, si lo que se pretende es, como creo, mostrar la estructura lógica del razonamiento en que debe fundarse la solución que, de acuerdo con las reglas relevantes, correspondería dar al caso - donde por "razonamiento" no se hace referencia a un proceso mental sino a una relación entre enunciados - lo que haya estado en la mente del juez es totalmente irrelevante (Zuleta, 2021, sección 5).

Supongo que es ahora evidente que esto no da en el blanco. En primer lugar, soy suficientemente claro en mi artículo (como ya mencioné en la sección 2.1) en que lo que considero que el silogismo jurídico intenta modelar no es "el proceso mental por el que el juez arriba a la decisión del caso", sino más bien la estructura lógica de la justificación de las decisiones que aplican derecho. Pero, en segundo lugar, dado que el silogismo jurídico pretende reconstruir adecuadamente los argumentos que los tribunales 
que aplican derecho suelen dar para justificar tales decisiones, "lo que haya estado en la mente del juez" tampoco puede ser "totalmente irrelevante", ya que si un determinado enunciado nunca pasó por la mente de un juez, y si no es un enunciado con el que pueda decirse que el juez se ha comprometido (aunque solo racionalmente) sobre la base de lo que sí pasó por su mente y de lo que dijo, entonces no puede figurar plausiblemente como premisa en una reconstrucción correcta de su argumentación.

\subsection{Zuleta sobre como reconstruir el argumento relevante}

Como parte de su discusión sobre el papel de las reglas universales, Zuleta ofrece en un dado momento una sugerencia, que también me gustaría discutir, sobre cómo reconstruir los argumentos judiciales relevantes. Expone su punto de vista de forma tentativa:

pienso que tal vez no sea necesario, a los fines de explicar el fundamento de una sentencia, que se formule completamente la regla tomada como premisa, sino que sería suficiente con mencionar aquellas propiedades que son relevantes para la subsunción del caso en consideración (Zuleta, 2021, sección 3.1).

Para ilustrar lo que quiere decir, considera dos disposiciones hipotéticas:

Artículo $k$ : Será penado con prisión de 8 a 25 años quien mate a otro/a.

Artículo l: No es punible quien mata a otro/a para defender su propia vida.

Si Pedro mata a Juan sin haber actuado en defensa propia, dice Zuleta, "la regla que justifica la condena de Pedro no requiere incluir en el antecedente la condición de que no haya matado en defensa propia, ya que la cuestión ni siquiera se planteó" (Zuleta, 2021, sección 3.1). El juez habrá aplicado "la regla: si alguien mata a otro[/a] debe ser condenado a prisión de 8 a 25 años". "Es cierto", continúa Zuleta, 
que si absuelve [un] acusado que mató en defensa de la propia vida el juez está interpretando que el artículo $l$ introduce una excepción a la regla derivada del artículo $k$, pero pienso que no era necesario mencionar la excepción al fundar lo resuelto en el primer caso (Zuleta, 2021, sección 3.1).

El punto de Zuleta es que "el hecho que el artículo $k$ tenga excepciones" es irrelevante, y no necesita ser mencionado, si "se trata de reconstruir el argumento en que se funda una decisión particular".

Creo que Zuleta tiene razón al presentar esta sugerencia solo de forma tentativa. No veo que pueda ser correcta. Y esto es porque, de nuevo, cualquier premisa que aparezca en la reconstrucción de un argumento es una premisa que consideramos que la persona que presentó el argumento tomó como verdadera. Pero en el escenario que describe Zuleta es manifiestamente falso que "si alguien mata a otro[/a] debe ser condenado a prisión de 8 a 25 años", si en efecto este enunciado de la norma debe interpretarse como especificando, en su antecedente, una condición suficiente del consecuente. Y eso significa que tal enunciado no es uno que podamos plausiblemente atribuir al tribunal como afirmación que el tribunal haya tomado como verdadera. Como dijo Glanville Williams, "una regla que está sujeta a una excepción es solo parcialmente verdadera si se enuncia sin la excepción" (Williams, 1988, p. 278).

Y si, por otro lado, Zuleta quiere decir que tal enunciado no debe entenderse como especificando una condición suficiente, sino de alguna otra manera - aunque nunca sugiere que sea esto lo quiere decir- entonces el argumento resultante ya no es, por supuesto, una instancia del silogismo jurídico; y queda por explicar cómo se desarrollaría la inferencia. ${ }^{6}$

6 Es cierto que la idea que motiva la sugerencia de Zuleta es intuitiva e incluso popular: es la idea de que los jueces no necesitan preocuparse por las excepciones en los casos en los que de hecho no se plantea ninguna excepción (es decir, que las excepciones importan solo en los casos en los que "la cuestión [...] se planteó"). Pero es solamente una idea pre-teórica y poco rigurosa y que no es fácil de articular con precisión. Lo he discutido también en Duarte d'Almeida, 2015a, pp. 162-168. 
Aplicación del derecho y la justificación de las decisiones judiciales

\subsection{Zuleta sobre el caso Luffe}

En "On the Legal Syllogism", discuto el caso $R v$ Luffe. Zuleta ofrece un útil resumen de este caso:

Se trata de una apelación en la que se discutía la competencia de los jueces de paz de cierto distrito para emitir una orden de filiación con relación al hijo adulterino de una mujer casada sobre la base de una disposición cuyo texto se refería a mujeres solteras. Para rechazar la apelación del demandado el tribunal argumentó que, a los fines de la disposición legal, merecía igual tratamiento el hijo adulterino que el nacido de una mujer soltera (Zuleta, 2021, sección 3.1).

Así es; y lo relevante es que al decidir en contra del recurrente el tribunal aplicó esa disposición. El caso Luffe es, escribí, "un ejemplo de una sentencia judicial real" que aplica derecho, pero "que la representación propuesta por el deductivismo de reglas es claramente incapaz de captar" (Duarte d'Almeida, 2019, p. 351).

Zuleta no considera que esto suponga ningún reto para el deductivismo de reglas. Da dos argumentos. El primero se refiere específicamente al caso Luffe. Zuleta no ve, dice, "qué razón puede haber para asegurar" que la disposición legal relevante fue efectivamente aplicada por el tribunal, "aparte del hecho de que fue esa la disposición mencionada en el fallo" (Zuleta, 2021, sección 3.1). Y yo mismo afirmo explícitamente, como señala Zuleta, que no creo que "cada vez que un tribunal afirma que aplica una disposición, se sigue de ello que nos encontramos ante una instancia de aplicación de esa disposición" (Duarte d'Almeida, 2019, p. 354). Pero entonces necesitamos saber "qué otros elementos deberíamos tener en cuenta para decidir la cuestión” (Zuleta, 2021, sección 3.1), y esto, dice Zuleta, es algo que yo no proporciono. De hecho, este es un punto que repite como una de sus principales conclusiones: que en mis artículos "no se presenta ningún criterio para distinguir las decisiones que aplican el derecho de las que no lo aplican" (Zuleta, 2021, sección 6).

7 Véase $R v$ Luffe (1807) 8 East 193; Duarte d’Almeida (2019, sección 4). 
Pienso que Zuleta se equivoca en todos los aspectos relevantes. Consideremos, en primer lugar, que, a diferencia de lo que afirma Zuleta, sí tenemos buenas razones para pensar en Luffe como un ejemplo de decisión que aplica derecho. ¿Cuáles son estas razones? En primer lugar, el hecho de que un tribunal lo diga es una de esas razones. Sostener, como hago, que la afirmación de un tribunal de que está aplicando una disposición no implica que efectivamente lo esté haciendo, no es decir que la afirmación del tribunal no sea buena evidencia de que sí está aplicando la ley. Y el tribunal de Luffe tiene claro que su decisión se basa en la afirmación de que el caso está "dentro de" ("within") la disposición legal relevante. ${ }^{8}$

Pero hay más: fue también así que la comunidad jurídica entendió y reportó aquella decisión. Véase el siguiente pasaje de un tratado contemporáneo, que caracteriza muy claramente a Luffe como una decisión en la que el tribunal aplicó la disposición pertinente:

Con relación a 18 Eliz. c. 3, que tiene las palabras "bastardos engendrados y nacidos fuera del matrimonio", y el 6 Geo. II., que solo se refiere al caso de una mujer soltera, se dudaba si estos preceptos se aplicaban si la madre era una mujer casada; pero finalmente se decidió [en $R v$ Luffe] que tenían esa aplicación (Theobald, 1836, p. 416).

Esto, de nuevo, es evidencia a favor de la afirmación de que el tribunal en el caso Luffe sí aplicó la disposición relevante. Es evidencia que ya había presentado en mi artículo ${ }^{9}$, aunque Zuleta no la mencione. Y, sobre todo, es evidencia del tipo correcto.

¿Qué quiero decir con "evidencia del tipo correcto"? El objeto de una caracterización teórica o filosófica de la aplicación del derecho (o de cualquier otra noción jurídica) es una noción de la cual abogados, tribunales e incluso ciudadanas y ciudadanos tienen ya una comprensión pre-teórica. Esta comprensión pre-teórica, que se manifiesta de varias maneras, es lo que habilita a los profesionales del derecho utilizar de forma competente la noción, e identificar, con confianza y correctamente, las instancias de 
aplicación del derecho. Proporciona también un importante punto de partida para la investigación teórica, y un criterio para evaluar la solidez de cualquier propuesta de explicación o caracterización de la noción.

Si un análisis o una teoría de la aplicación del derecho no capta o no da cuenta de los casos que la propia comunidad jurídica consideraría como casos de aplicación del derecho, eso cuenta en contra de la teoría. Es un coste. Esto no quiere decir que sea un coste fatal: por supuesto, puede haber espacio para que el teórico desestime como erróneos algunos de los juicios pre-teóricos de los profesionales del derecho. Pero esas desestimaciones tienen que estar respaldadas por la teoría; la teoría tiene que ser capaz de explicar ("explain away") esos juicios si los considera erróneos.

Lo que el teórico no puede hacer es simplemente descartar el desajuste entre su explicación de la aplicación del derecho y la comprensión pre-teórica de los juristas. Tampoco puede el teórico (ni nadie) decir simplemente que, a menos que el jurista sea capaz de proporcionar un criterio para distinguir los casos en los que los tribunales aplican el derecho, pero lo hacen de forma incorrecta, de los casos en los que el tribunal no aplica el derecho, no es necesario tener en cuenta la propia comprensión del jurista. Y esto es porque, al fin y al cabo, proporcionar ese criterio es precisamente parte de lo que la teoría debe proporcionar. Es tarea de los teóricos del derecho - no de los juristas o de los profesionales del derecho- articular tales criterios.

Por lo tanto, Zuleta se equivoca al descartar como irrelevante la fuerte evidencia - proporcionada por la opinión explícita de la comunidad jurídica - de que en una decisión como la de Luffe un tribunal sí aplica derecho. ${ }^{10} \mathrm{Y}$ si, a la vista de esa evidencia, Zuleta sigue pensando que Luffe no es un ejemplo de decisión que aplica derecho, entonces la carga de demostrarlo recae en él. Por supuesto, no lo intenta. Y no creo que sea capaz de hacerlo sin asumir la corrección de una teoría como el deductivismo de reglas: pero entonces estaría incurriendo en petitio principii.

Y Zuleta también se equivoca al afirmar que en mis artículos no "suministr[o] criterios para distinguir aquellos casos en que una disposición fue mal aplicada de aquellos en que simplemente no fue aplicada" (Zuleta,

10 Tampoco Luffe es un ejemplo aislado de decisiones de este tipo: véase la discusión en Duarte d'Almeida (2019, sección 4.B). 
2021, sección 3.1). Por supuesto que lo hago. Proporcionar tales criterios, como acabo de decir, es la tarea de una teoría de la aplicación del derecho - y es precisamente lo que proporciona mi propia caracterización teórica de la aplicación del derecho-.

¿Cuál es entonces mi criterio? Es muy sencillo de exponer. Un tribunal aplica el derecho - lo aplica pragmáticamente- cuando su decisión se ajusta a mi definición, y no lo hace en el caso contrario. Y un tribunal aplica mal el derecho siempre que cualquiera de los enunciados relevantes que la definición menciona resulte ser falso: cuando, por ejemplo, la decisión que el tribunal considera como la acción que debería realizar sobre la base de la disposición relevante no sea en realidad la acción que debería realizar sobre esa base; o cuando el juicio de deber relevante no sea, de hecho, apoyado normativamente (aunque el tribunal lo considere así) en la conclusión de la aplicación inferencial de la disposición relevante.

Este es, pues, mi criterio: y la decisión del caso Luffe lo satisface.

\section{7. ¿Es el deductivismo de reglas inmune a contraejemplos?}

He dicho al inicio de la sección anterior que Zuleta tiene dos argumentos contra mi sugerencia de que un caso como Luffe plantea un desafío al deductivismo de reglas. Su segundo argumento es más general.

Zuleta piensa que una teoría como el deductivismo de reglas no necesita dar cuenta de "todos los contraejemplos en que en el uso común de los juristas se considera que se ha aplicado una disposición y que no encajan claramente en su modelo" (Zuleta, 2021, sección 3.1). Y a este respecto hace dos observaciones relacionadas, apoyándose en Carnap para la primera, y en Hempel para la segunda.

El primer punto es que, "como señala Carnap", el objetivo de una elucidación es "sustituir un concepto dado más o menos inexacto, el explicandum, por otro exacto, el explicatum" (Zuleta, 2021, sección 3.1). Ya que "el explicatum debe ser tan exacto como sea posible, en tanto que el explicandum es vago, no es posible esperar una coincidencia total", $\mathrm{e}$ 
incluso pueden aceptarse apartamientos considerables, como en el caso del concepto científico de "pez", que excluye de su alcance a algunas especies que eran consideradas peces en el uso común, como las ballenas y las focas (Zuleta, 2021, sección 3.1).

Por supuesto. Pero — dejando a un lado que no está claro que lo que una teoría como el deductivismo de reglas pretende ofrecer sea algo como una explicación carnapiana-Carnap es muy claro en cuanto a que la similitud entre un explicatum y su explicandum es un requisito para una explicación bien sucedida, y que las pérdidas de similitud deben justificarse por las ganancias en exactitud y fecundidad (Carnap, 1962, pp. 5-8).

Esto es ciertamente así para el concepto científico Pez, que es, como escribe Carnap, "mucho más estricto" que el concepto pre-científico, ya que excluye muchos tipos de animales que este incluía (Carnap, 1962, p. 6). Pero esta exclusión - con el correspondiente "cambio en las reglas del lenguaje" - fue motivada por "descubrimientos fácticos" (Carnap, 1962, p. 6) relevantes, y se justifica dentro del contexto más amplio de "la construcción de un lenguaje sistemático de la zoología” (Carnap, 1962, p. 5). No es que los zoólogos se limitaran a descartar las ballenas y las focas como "no peces" y no tuvieran nada más que decir sobre ellas. Su exclusión del concepto científico Pez va acompañada de su inclusión en diferentes categorías que forman parte del mismo esfuerzo de sistematización coherente.

Pero nada de esto es cierto en el caso del deductivismo de reglas. Los deductivistas de reglas no afirman que el coste de no captar todos los casos de lo que los profesionales del derecho consideran aplicación del derecho esté justificado por la particular fecundidad o exactitud del modelo que proponen. Zuleta tampoco hace tales reivindicaciones en nombre de los deductivistas de reglas. Lo hace parecer como si cualquier disimilitud entre un explicatum y su explicandum pudiera simplemente descartase señalando que el requisito carnapiano de similitud no es absoluto. Ojalá las cosas fueran tan sencillas.

Para su segundo punto, que está relacionado con el anterior, Zuleta recurre a Hempel. Dice que "el modelo deductivista de reglas es estructuralmente igual a otros modelos de explicación por subsunción legal”, y que "más precisamente, el silogismo jurídico tiene la misma estructura 
que la explicación científica en el modelo lógico-deductivo" (Zuleta, 2021, sección 3.1). Y señala que Hempel dice que tales modelos de explicación "no pretenden describir la manera como los científicos formulan realmente sus descripciones explicativas" (Zuleta, 2021, sección 3.1). Su "propósito", escribe Hempel, "es más bien indicar en términos razonablemente precisos la estructura lógica y la justificación de diversos modos en que la ciencia empírica responde a las preguntas que piden una explicación" (Hempel, 1979, p. 405). Por tanto, dice Hempel, "no tiene objeto quejarse de que los modelos de leyes inclusivas no se ajustan exactamente a la forma en que los científicos presentan realmente sus explicaciones" (Hempel, 1979, p. 420).

Mi respuesta, de nuevo: por supuesto. Pero Zuleta vuelve a errar el blanco. El punto ahora, aplicado a nuestro tema, es sobre la diferencia entre el silogismo jurídico como modelo de la justificación de las decisiones que aplican derecho, y la manera informal en que los tribunales realmente presentan sus propias justificaciones para tales decisiones. Pero este es un punto que no solo planteo yo mismo en "On the Legal Syllogism", sino uno en que me detengo extensamente. Es el punto - que he mencionado también en la sección 2.1 - sobre la diferencia entre una reconstrucción de un argumento y la manera en que el argumento es de hecho presentado por el tribunal. Y este punto no nos dice nada sobre cuán problemático para el deductivismo de reglas es un contraejemplo como Luffe. El punto de un ejemplo como Luffe no es que el tribunal no presenta su argumento en la forma del silogismo jurídico, sino que ese argumento no es reconstruible como un silogismo jurídico.

Zuleta concluye su análisis de estas cuestiones diciendo que

las observaciones de Hempel son aplicables, mutatis mutandis, al modelo del deductivista de reglas. Por ello, aun cuando su noción de aplicación de reglas no cubra todos los supuestos en que los juristas afirman que se ha aplicado una disposición, esto no es una razón suficiente para descartarla, a menos que se ofrezca una noción alternativa que, además de ser más cercana al uso común, sea al menos tan exacta y fértil como aquélla que se pretende reemplazar (Zuleta, 2021, sección 3.1). 
En respuesta cabe hacer tres puntos. El primero es que Zuleta entiende las cosas exactamente al revés. Si el deductivismo de reglas no da cuenta de toda la gama de su explicandum, entonces eso, como vimos anteriormente, es un costo que el propio deductivismo de reglas tiene que ser capaz de justificar. En segundo lugar, yo ofrezco en mis artículos un modelo que da cuenta de todos los casos que el deductivismo de reglas tiene en cuenta $y$ de los casos que no tiene; y no veo, y Zuleta no argumenta, que mi modelo sea menos exacto o fructífero que el de los deductivistas de reglas.

En tercer lugar, y más importante: a diferencia de lo que sugiere Zuleta, yo no concedo que el deductivismo de reglas sea capaz de dar cuenta satisfactoriamente de muchos o incluso algunos casos de decisiones judiciales que aplican derecho, aunque haya casos, como Luffe, que no logra captar. Más bien, yo utilizo Luffe como simple medio para poner más claramente de manifiesto las deficiencias generales del deductivismo de reglas. Y lo digo explícitamente en "On the Legal Syllogism", inmediatamente antes de introducir el caso Luffe:

El modelo del silogismo es, en mi opinión, inadecuado incluso en los casos más fáciles, cuando no hay duda de que la regla relevante se aplica al caso en cuestión. Esos son casos que el modelo solo parece captar bien; la apariencia es engañosa. Pero los problemas con el modelo son más fácil y efectivamente exhibidos si prestamos atención a un ejemplo de sentencia judicial real que la representación propuesta por el deductivismo de reglas es claramente incapaz de captar (Duarte d'Almeida, 2019, p. 351).

\subsection{El cambio de tema de Zuleta}

Zuleta tiene un último argumento que plantear en relación con mi crítica al deductivismo de reglas. Como ya mencioné en la sección 2.1, escribe Zuleta que "el principal problema" que encuentro en el silogismo jurídico es "que en ningún lugar se dice que la disposición legal se aplica al caso en consideración" (Zuleta, 2021, sección 1). Y aunque está de acuerdo (por supuesto) en que esto es cierto, dice que "no es claro por qué debería expresarse esa idea en las premisas" (Zuleta, 2021, sección 3.2). Argumenta lo siguiente: 
Lo que la reconstrucción del deductivista de reglas esquematiza es la relación lógica que debe haber entre una regla y el contenido conceptual de la decisión para que se pueda sostener que la regla se aplicó a un caso o que la decisión tiene fundamento en la regla. La aplicación de la regla no se afirma sino que se muestra. En este punto, la objeción de Duarte d'Almeida parece asemejarse a la de quien criticara el teorema de Pitágoras sobre la base de que en ninguna premisa se afirma que los principios de Euclides son aplicables a los triángulos rectángulos (Zuleta, 2021, sección 3.2).

Pero esto, además de poco caritativo, no viene al caso. Zuleta se equivoca al decir que el hecho de que el silogismo jurídico no incluya, en sus premisas, la afirmación de que la disposición pertinente es aplicable, constituye, para mí, un "problema” - "principal” o no- del deductivismo de reglas. El "principal” problema del deductivismo de reglas (si se quiere ordenarlos), más bien, es que el modelo del silogismo atribuye a los jueces compromisos con premisas que no es plausible considerar que ellos acepten.

De hecho, solo empiezo a exponer mis argumentos sobre los enunciados de aplicabilidad (como enunciados de segundo orden) y su papel argumentativo en la última sección de "On the Legal Syllogism" — ya después de haber concluido mi crítica del deductivismo de reglas y de haber concluido que necesitamos un modelo alternativo (Duarte d'Almeida, 2019, p. 359)—. Que el silogismo jurídico "no expresa que la disposición se aplique al caso" no es una de las razones que ofrezco contra el deductivismo de reglas.

Más bien, lo que sugiero es que un modelo satisfactorio de la justificación de las decisiones que aplican derecho — que es algo que el silogismo jurídico en cualquier caso no proporciona - debería incluir, como premisas, enunciados de segundo orden sobre aplicabilidad, en lugar de enunciados de primer orden sobre las "reglas" putativamente relevantes. Justifico esta sugerencia con argumentos que Zuleta no aborda o siquiera menciona.

Pero esto forma ya parte de mis propias ideas sobre cuál sea el modelo correcto, no de mi crítica al modelo deductivista de reglas; y Zuleta también tiene algunas observaciones críticas que hacer al respecto de cómo entiendo la noción de aplicabilidad. 


\subsection{Zuleta sobre el modelo de la aplicabilidad}

Cuando pasa a discutir las ideas que defiendo en “¿Qué es aplicar derecho?”, Zuleta se centra en lo que tengo para decir sobre la noción de aplicabilidad. Cita mi definición, según la cual

decir que una disposición $p$ se aplica a un objeto $x$ es decir que hay una consecuencia jurídica específica (o consecuencias jurídicas específicas) que la existencia misma de $p$ tiene para $x$ como cuestión de derecho. Es, por lo tanto, decir que hay una afirmación específica sobre $x$ que es verdadera como cuestión de derecho, y que no podría, dada $p$, ser falsa (Duarte d'Almeida, 2021, Sección 2.3).

Zuleta no cree que mi definición, junto con mis comentarios explicativos sobre ella, "suministren una idea, siquiera aproximada, de lo que significa que una disposición es aplicable a un objeto" (Zuleta, 2021, sección 4), y le parece extraño que en mi propuesta de reconstrucción del argumento que justifica una decisión judicial que aplica derecho haya una premisa que simplemente afirma "que la disposición es aplicable al objeto" sin que se sepa por qué (Zuleta, 2021, sección 4). Y esto socava, sugiere Zuleta en una de sus conclusiones, mi propia caracterización de la aplicación inferencial del derecho (Zuleta, 2021, sección 6).

Pero Zuleta malinterpreta lo que digo. Parece pensar que cuando hablo de la consecuencia (o consecuencias) jurídica que la disposición tiene para un determinado objeto, estoy hablando de consecuencias lógicas. Su discusión del ejemplo de una disposición que dice "está permitido contraer matrimonio si y solo si ambos contrayentes son solteros" y de las consecuencias lógicas de la conjunción de esa disposición con ciertas proposiciones así lo sugiere (Zuleta, 2021, sección 4).

Sin embargo, por "consecuencia" yo no me refiero a una consecuencia lógica. De hecho, las disposiciones jurídicas ni siquiera son el tipo de cosas que pueden servir como relata lógicos. Las disposiciones en sí mismas no tienen valores de verdad, sino que existen o no como parte del derecho válido. Por supuesto que una disposición — un artículo de una ley, por ejemplo- puede ser redactada, como en el ejemplo de Zuleta, en una 
oración que podríamos considerar que tiene valor de verdad. Pero las consecuencias jurídicas de una disposición no son las consecuencias lógicas de cualquier oración que se haya utilizado en su formulación. Y cuando caracterizo la noción de aplicabilidad, soy explícito (como puede leerse en el pasaje recién citado) que lo que está en juego es la consecuencia (o consecuencias) jurídica(s) que "la existencia misma" de la disposición tiene para un determinado objeto.

¿Qué entiendo entonces por las consecuencias de una disposición? Me refiero a los efectos jurídicos que se le pueden atribuir en relación con un caso particular que se tenga enfrente. En el ejemplo de The Hollandia - mi principal ejemplo en “¿Qué es aplicar derecho?”-, la disposición pertinente, el artículo III, párrafo 8, de las normas de La Haya-Visby, tuvo como consecuencia, según Lord Denning, la nulidad de la cláusula del conocimiento de embarque en cuestión. Y esto no es, como también advierto explícitamente, una cuestión sobre el lenguaje de la disposición; no se trata de lo que la disposición dice. Y tampoco se trata de la consecuencia que la disposición podría tener para otras cláusulas de conocimientos de embarque en otros casos particulares. Se trata, más bien, de la consecuencia concreta que la disposición tiene para aquel conocimiento de embarque en particular.

Por eso digo que "la aplicación del derecho es, en cierto sentido, un asunto particularista" (Duarte d'Almeida, 2021, sección 2.4). Zuleta también se opone a esta afirmación. “Teniendo en cuenta que el particularismo es lo opuesto a la aplicación de normas generales y que el derecho está compuesto esencialmente por normas generales", escribe, "la afirmación no me parece inteligible" (Zuleta, 2021, sección 4).

Bien, yo esperaba que mi uso de la frase "en cierto sentido" se entendiera a la luz de mis explicaciones precedentes —incluyendo, como ya he mencionado, mis puntos sobre la universalidad de todos los juicios normativos particulares-. Pero en un punto tiene Zuleta razón: yo no formularía mi visión del derecho diciendo que "el derecho está compuesto esencialmente por normas generales" - al menos si estas "normas generales" son las que se supone que los jueces aplican-. Pero este no es el lugar para profundizar este asunto. Mis dos artículos sobre la justificación de las decisiones judiciales — uno sobre el deductivismo de reglas, el otro sobre 
la aplicación del derecho- son solo dos partes de una visión más amplia del derecho en general (y de la actividad que comúnmente se denomina “interpretación” jurídica) que espero seguir desarrollando en posteriores trabajos.

En cualquier caso, Zuleta no es el único de mis comentaristas que se centra en mi afirmación de que la aplicación del derecho es "un asunto particularista”. Gabriela Scataglini, en particular, cuestiona la conexión entre esa afirmación y mi discusión sobre el deductivismo de reglas: cree que no hay ninguna. Pasemos ahora a sus observaciones.

\section{Respuesta a Gabriela Scataglini}

Scataglini, al igual que Zuleta, discute no solo los puntos de vista que presento en “¿Qué es aplicar derecho?”, sino también algunos aspectos de mis críticas al deductivismo de reglas en "On the Legal Syllogism". Y aunque está de acuerdo conmigo en un punto central de esas críticas, no está segura de que yo haya explicado bien cómo se conectan entre sí la pars destruens y la pars construens de mi propuesta.

Scataglini discute mis ideas en tres frentes. Trataré cada uno de sus puntos por separado, en las secciones 3.1, 3.3 y 3.4. Pero también aprovecharé la oportunidad para mostrar, en la sección 3.2., que mientras tenemos buenas razones para rechazar el deductivismo de reglas, los propios deductivistas de reglas no tienen ninguna razón para rechazar mi propuesta.

\subsection{Scataglini sobre argumentos, premisas, y enunciados de primer y segundo orden}

En la primera sección de su artículo, Scataglini parece atribuirme la tesis de que, cuando los jueces justifican sus decisiones de aplicación del derecho, la conclusión de sus argumentos es la propia decisión. Presenta mis puntos de vista sobre el silogismo jurídico como si mi opinión fuera que el problema de este modelo es que, dadas sus premisas, "la conclusión del razonamiento no resulta ser la decisión judicial (sentencia)”. También 
sugiere que mi propio modelo representa el tipo de argumento necesario "para poder derivar lógicamente un enunciado que constituya una sentencia o decisión judicial” (Scataglini, 2021, sección 1).

Esto es incorrecto. No creo que la conclusión del argumento relevante sea la propia decisión que aplica el derecho, ni que lo que el silogismo jurídico pretenda reconstruir sea "un razonamiento cuya conclusión es la decisión judicial (sentencia)”. Una decisión es una acción, mientras que la conclusión del argumento relevante es una frase o proposición dotada de valor de verdad; y las acciones no son frases o proposiciones. Este es, de hecho, un punto que hago explícitamente en "On the Legal Syllogism" (Duarte d'Almeida, 2019, p. 350).

Lo que sí digo es que, si el argumento relevante está destinado a justificar la decisión, entonces la frase que figura como su conclusión debería aseverar que esa decisión es una que el juez debe jurídicamente, todo considerado, emitir (o al menos una decisión que, todo considerado, le está jurídicamente permitido emitir). Y, al discutir el modelo del silogismo jurídico, lo considero en primer lugar (en la sección III de "On the Legal Syllogism") bajo el supuesto de que ese es el tipo de conclusión que las premisas pertinentes pretenden justificar (Duarte d'Almeida, 2019, pp. 346-350). Pero luego - habiendo concluido que el silogismo jurídico es incapaz de modelar eso- procedo a considerarlo (en la sección IV) como un modelo de justificación de proposiciones jurídicas particulares, y muestro cómo el modelo del silogismo jurídico falla también cuando es reinterpretado de esa manera (Duarte d'Almeida, 2019, pp. 350-359).

Pero lo interesante de las observaciones de Scataglini en la primera sección de su artículo radica en la analogía que establece entre, por un lado, mi afirmación de que un modelo satisfactorio de la justificación de las decisiones que aplican derecho debería incluir, como una de las premisas, un enunciado de aplicabilidad, que es un enunciado de segundo orden (en lugar de un enunciado de primer orden de una regla), y, por otro lado, lo que parece ser la posición de la Tortuga en el conocido diálogo de Lewis Carroll entre Aquiles y la Tortuga (Carroll, 1895).

No es necesario que repita la presentación que hace Scataglini del diálogo. Lo que importa acá es su punto sobre "la enseñanza principal del relato de Carroll": a saber, que "la hipótesis -i. e., el condicional que la 
Aplicación del derecho y la justificación de las decisiones judiciales

Tortuga debe 'aceptar' para pasar a la conclusión - no debe ser tratada como una premisa más porque constituye un enunciado de segundo orden" (Scataglini, 2021, sección 1).

La analogía, dice Scataglini, está en que yo parezco

partir de la misma intuición que tiene la Tortuga: que las [...] premisas de primer orden no llevan - no obligan - a aceptar la conclusión. Para afirmar la conclusión se necesita hacer algo más: algo que solo puede ser captado (reconstruido) en un orden superior (Scataglini, 2021, sección 1).

Y la preocupación para mí sería que - como el diálogo de Carroll se supone que enseña- los "enunciados de segundo orden no pueden aparecer 'mezclados' en el mismo razonamiento con las premisas de primer orden" (Scataglini, 2021, sección 1).

Me parece, sin embargo, que no hay ninguna analogía relevante, y que Scataglini incurre en un equívoco respecto a la noción de enunciado de "segundo orden".

Obsérvese, en primer lugar, que se trata de una noción relacional. Un enunciado solo es apropiadamente llamado "de segundo orden" en relación a algún otro enunciado o noción que mencione. Así, en el diálogo de Carroll, el enunciado "Si A y B son verdaderas, entonces $Z$ debe ser verdadera" es un enunciado de segundo orden en relación a los enunciados que menciona - A, B y Z- que son, en consecuencia, de "primer orden" (Scataglini, 2021, sección 1).

Nótese también que en el diálogo de Carroll la cuestión, como dice Scataglini, es que aquel enunciado de segundo orden, que puede tomarse como expresión de un "principio de inferencia", no debe añadirse a A, B y $\mathrm{Z}$ como una premisa más del argumento. Esta es, según Scataglini, la forma adecuada de resolver la paradoja planteada por la Tortuga.

Pero mis objeciones al silogismo jurídico no se parecen en nada a las de la Tortuga. Para empezar, no niego - por el contrario- que el silogismo jurídico sea el esquema de una inferencia lógicamente válida. De hecho, así lo afirmo (Duarte d'Almeida, 2019, p. 339). Así que mi punto no tiene nada que ver con la cuestión de si sus premisas apoyan la conclusión. Mi punto, más bien (como también he explicado en la Sección 2.2) es que la premisa 
jurídica no puede atribuirse plausiblemente a los jueces cuyos argumentos el silogismo jurídico pretende reconstruir.

Y cuando en mi propia propuesta incluyo, como premisas, lo que denomino enunciados de aplicabilidad, y digo que se trata de enunciados de "segundo orden", lo que quiero decir es simplemente que se trata de enunciados acerca de las disposiciones pertinentes - es en ese sentido que son de "segundo orden" - y no de enunciados del contenido de las reglas que tales disposiciones supuestamente expresan. Sin embargo, como premisas en los argumentos pertinentes, los enunciados de aplicabilidad son, lógicamente hablando, enunciados de "primer orden" precisamente en el mismo sentido en que los enunciados A y B del diálogo de Carroll son enunciados de "primer orden". Son las premisas relevantes que, dada la regla de inferencia apropiada (que en sí misma, estoy de acuerdo, no es una premisa, y por tanto es lógicamente un elemento de "segundo orden"), conllevan la conclusión.

Puedo plantear el mismo punto de otra manera. Tomemos cualquier enunciado de "segundo orden". Por supuesto, es perfectamente posible construir un argumento lógicamente válido que incluya ese enunciado como una de sus premisas. En el contexto de ese argumento, entonces, ese enunciado será lógicamente un enunciado de primer orden.

Pero como mi uso de la expresión "de segundo orden" para referirme a los enunciados de aplicabilidad parece haber provocado cierta confusión, tal vez sea ahora un buen momento para hacer algunas observaciones básicas sobre el modelo que he propuesto y sobre lo que los deductivistas de reglas pueden decir sobre él.

\subsection{Por qué incluso los deductivistas de reglas podrían aceptar el modelo de la aplicabilidad}

Cuatro puntos simples - casi trivialidades, diría-.

En primer lugar, no hay nada inusual en lo que llamo "enunciados de aplicabilidad" (es decir, enunciados de la forma, por ejemplo, "el artículo $A$ se aplica a este contrato"). La etiqueta es mía, pero los enunciados de 
Aplicación del derecho y la justificación de las decisiones judiciales

aplicabilidad son comunes en la práctica y el lenguaje de los tribunales y abogados. ${ }^{11}$

En segundo lugar, los deductivistas de reglas (y cualquier persona, en realidad) podrían y deberían estar de acuerdo con la afirmación de que si un determinado enunciado de aplicabilidad es verdadero - si, por ejemplo, es verdadero que un determinado artículo de una ley se aplica a un determinado objeto- entonces, como resultado, habrá algún enunciado jurídico particular sobre ese objeto que será verdadero también. En The Hollandia - mi principal ejemplo en “¿Qué es aplicar derecho?”-, la aplicabilidad del artículo III, párrafo 8, de las reglas de La Haya-Visby a la cláusula pertinente del conocimiento de embarque tiene como resultado que es verdadero, como cuestión de derecho, que esa cláusula es nula.

En tercer lugar, los deductivistas de reglas (o, de nuevo, cualquier otra persona) no tienen ninguna razón para negar que, cuando tal enunciado particular es verdadero, eso da al tribunal una razón para decidir el caso de una manera determinada (a saber, en el ejemplo de The Hollandia, permitiendo la apelación).

Y, en cuarto lugar, si un tribunal tiene tal razón para decidir de una manera determinada, entonces esa es la decisión que, todo considerado, debe emitir jurídicamente si esa razón no se ve superada por ninguna consideración compensatoria, es decir, si no hay consideraciones de igual o mayor fuerza en sentido contrario.

Considero que estos cuatro puntos son trivialmente verdaderos; no son puntos que los deductivistas de reglas deban negar. ${ }^{12}$ Pero si son ver-

${ }^{11}$ Señalo en la sección 2.5 de “QQue es aplicar derecho?” que la disposición puede ser aplicada a "cualquier objeto" $x$. Scataglini se pregunta, en sección 1 de su comentario, sobre casos en los que lo que está en juego no es un "objeto", sino un evento - "un hecho (como ser, por ejemplo, una acción llevada a cabo por una persona)"-. Pero la pregunta está mal planteada. Mi uso de la palabra "objeto" no pretende referirse únicamente a los objetos materiales. Más bien, utilizo "objeto" en el mismo sentido en que se habla, por ejemplo, del "objeto del discurso", o del "objeto" de una teoría: lo utilizo simplemente para referirme a cualquier cosa a la que se aplique la disposición. Puede ser una acción, una persona, o de hecho cualquier otra cosa.

12 Estos cuatro puntos reiteran, de forma simplificada pero también actualizada — a la luz de lo que digo en “¿Qué es aplicar derecho?” (Duarte d’Almeida, 2021) —, lo que expuse originalmente en la última sección de "On the Legal Syllogism" (Duarte d’Almeida, 2019, pp. 359-360). 
daderos, esto significa que el siguiente esquema - que reúne los cuatro puntos- bien podría captar un argumento que cualquier tribunal podría dar para justificar una decisión $\varphi$ sobre la base de algún enunciado jurídico particular $c$ que es en sí mismo el producto de una cierta disposición $p$ que se aplica a algún objeto $x$ :

(1) Si la disposición $p$ se aplica a $x$, entonces $c$.

(2) La disposición $p$ se aplica a $x$.

Por lo tanto (de (1) y (2)),

(3) $c$.

(4) $\mathrm{Si} c$, y no hay consideraciones compensatorias [i.e. consideraciones de igual o mayor fuerza en sentido contrario], entonces, todo considerado, este tribunal debe jurídicamente (o no es el caso que no deba jurídicamente) $\varphi$.

(5) No hay consideraciones compensatorias.

Por lo tanto (de (3), (4) y (5))

(6) Todo considerado, este tribunal debe jurídicamente (o no es el caso que no deba jurídicamente) $\varphi$.

Y este es, por supuesto, el esquema que propongo para la justificación de las decisiones que aplican derecho: el esquema que presenté anteriormente, en la sección 2.3. A diferencia del silogismo jurídico de los deductivistas de reglas, no incluye ningún enunciado de una regla como premisa. Pero si - como acabo de decir - no hay nada en el esquema que he propuesto a lo que los deductivistas de reglas se opondrían, ¿por qué exactamente no podrían aceptarlo como un modelo satisfactorio?

La respuesta, como sabemos, es que los deductivistas de reglas tienen una comprensión particular de lo que significan los enunciados de aplicabilidad. Desde su punto de vista, la premisa (1) es sinónimo de una afirmación más o menos del siguiente tenor: "el antecedente de la regla universal expresada por el artículo $X$ es satisfecho por $Y$ ".

Pero nótese que esto también es un enunciado de "segundo orden". Es un enunciado sobre la regla y su relación con $Y$. Lo que significa que, si los deductivistas de reglas tuvieran razón sobre este punto, podrían adoptar el modelo que propongo, con la única diferencia de que la premisa (1) ten- 
dría que reescribirse como la afirmación de que "el antecedente de la regla expresada por el artículo X se satisface con Y”. Lo que quiero decir es que no hay nada en la idea de que el argumento relevante pueda reconstruirse con tales enunciados de "segundo orden" que los deductivistas de reglas tengan alguna razón para negar.

El problema es que los deductivistas de reglas -incorrectamente, en mi opinión - también considerarían que esto justifica su afirmación de que el argumento pertinente podría ser reformulado en la forma del silogismo jurídico, un argumento que atribuye al juez un compromiso con los enunciados de primer orden de las reglas correspondientes. ¿Y por qué es esto un problema? Porque la afirmación "el antecedente de la regla expresada por la disposición $p$ se satisface con $x$ ” no es sinónima de -ni implica- ningún enunciado real de la regla universal expresada por $p, y$ porque es muy posible creer justificadamente esa primera afirmación sin una opinión sobre cómo debe formularse la regla universal expresada por la disposición $p$.

$\mathrm{Y}$ es por eso que sugiero que nos ciñamos a los enunciados de aplicabilidad de "segundo orden" (aunque no propongo entenderlos como lo harían los deductivistas de reglas, por supuesto) en nuestra reconstrucción del argumento relevante.

También espero que ahora esté más claro que no hay nada particularmente extravagante en la reconstrucción que propongo.

\subsection{Scataglini sobre el carácter particularista de la aplicación del derecho}

¿Cuál es exactamente la conexión, se pregunta Scataglini al final de la sección 1 de su artículo, entre mi crítica al silogismo jurídico y mi observación de que considero que la aplicación del derecho es "en cierto sentido, un asunto particularista"?

Scataglini dice que no "alcanz[a] a ver [...] cuál sería exactamente el vínculo entre ambos aspectos” (Scataglini, 2021, sección 1). Pero eso se debe a que ha incorrectamente tomado mi planteamiento sobre los enunciados de aplicabilidad de "segundo orden" como si reflejara las quejas de la Tortuga en 
el diálogo de Carroll. El vínculo, como podemos ver ahora, es directo: el silogismo jurídico atribuye injustificadamente a los tribunales un compromiso con enunciados de reglas universales, mientras que mi esquema les atribuye solo un compromiso con los enunciados de aplicabilidad particulares. Los enunciados de aplicabilidad particulares - que, de nuevo, los deductivistas de reglas no tienen razón para negar que los tribunales pueden dar, como a menudo lo hacen - no son sinónimos de enunciados de reglas universales, y pueden sostenerse por sí mismos sin apelar a ellos.

Y en el tema del particularismo, así entendido, Scataglini está de acuerdo conmigo, y de hecho ha defendido puntos de vista similares en sus propios trabajos. Scataglini "coincide", dice, con mi idea "de que aplicar normas jurídicas no consiste en la representación ni el compromiso por parte del agente de una proposición universal conteniendo todas las aplicaciones de la norma" (Scataglini, 2021, sección 2) y subraya, de manera importante, que esto:

no supone negar que existen criterios de corrección acerca de si una norma es o no aplicable a un caso particular. De hecho, un juez o jueza ponen en uso ese criterio cuando aplican una norma a un caso. Pero lo que no hacen es captar o comprometerse con una fórmula o principio universal que contiene (extensional o intensionalmente) todas las aplicaciones de la norma (Scataglini, 2021, sección 2).

Debo decir que no estoy totalmente de acuerdo con todos los detalles de cómo Scataglini presenta sus propios puntos de vista sobre el asunto. Scataglini habla de jueces que aplican las normas pertinentes, mientras que yo hablo de jueces que aplican disposiciones, por ejemplo. También establece conexiones entre la noción de aplicabilidad y la idea de subsunción que yo no suscribiría necesariamente.

Pero no es necesario ahora dedicar tiempo a estos puntos de desacuerdo relativamente menores. Dada la similitud general entre sus puntos de vista y los míos sobre esta cuestión, supongo que Scataglini también debe encontrar el modelo del silogismo inadecuado como representación de la justificación de las decisiones judiciales. No lo dice explícitamente, pero debería hacerlo. 


\subsection{La conexión normativa entre la aplicación inferencial y la aplicación pragmática del derecho}

En la última sección de su trabajo, Scataglini se centra en la conexión entre la aplicación inferencial y pragmática del derecho, y en particular en mi afirmación de que "la aplicación pragmática del derecho [...] retroalimenta normativamente la aplicación inferencial del derecho" (Duarte d'Almeida, 2021, sección 4).

Scataglini expresa sus dudas al respecto. Parece estar de acuerdo con mi planteamiento de que, al considerar si una determinada disposición se aplica a un determinado objeto, un tribunal debería estar atento a las consecuencias que se derivarían -incluso para la manera en que el tribunal debería decidir el caso- si un enunciado de aplicabilidad particular se considerara verdadero. Pero entonces, sugiere que me equivoco al pensar que "la evaluación de las consideraciones compensatorias corresponde exclusivamente a la aplicación pragmática” (Scataglini, 2021, sección 3). ${ }^{13}$

La observación de Scataglini es acertada, y me hace ver que podría haber sido más claro en lo que quiero decir. Pero intentaré ahora explicar mis puntos con más precisión.

Lo primero es que hay una serie de consideraciones - de razones - que influyen en la cuestión de si una disposición $p$ se aplica, con una consecuencia particular, a un objeto particular $x$. Estas razones incluyen, creo -y lo digo en “¿Qué es aplicar derecho?”-, las consideraciones comúnmente contempladas bajo el epígrafe de "interpretación jurídica" (Duarte d’Almeida, 2021, sección 2.4). Y estoy de acuerdo con Scataglini en que puede haber razones tanto a favor como en contra de un determinado enunciado de aplicabilidad, y, por tanto, que cualquier razón a favor solo determinará al enunciado de aplicabilidad relevante si no hay consideraciones de igual o mayor fuerza en sentido contrario.

${ }^{13}$ Obsérvese también que esto no es sustancialmente diferente de lo que dice Simpson en la cita que di más arriba (en la sección 2.2) al discutir algunas de las críticas de Zuleta: "una persona puede actuar en base a una norma sin pensar en el bosquejo de una norma" (Simpson, 1961, p. 162). 
Por tanto, estoy de acuerdo con Scataglini cuando escribe que a la hora de determinar si una disposición se aplica de forma inferencial, con una determinada consecuencia, a un determinado objeto, "evaluamos posibles consideraciones compensatorias y sostenemos nuestras conclusiones como ajustadas al derecho, como la determinación correcta de lo que el derecho dispone" (Scataglini, 2021, sección 3). Y también estoy de acuerdo en que esto es algo que hacen no solo los jueces, sino los "abogados y abogadas litigantes", los “juristas o quienes se dedican a la dogmática”, y más generalmente las ciudadanas y ciudadanos. (Como menciono en mi artículo, "a menudo, como meras y meros ciudadanas y ciudadanos, sí aplicamos inferencialmente el derecho”, Duarte d’Almeida, 2021, sección 3.5.) No pretendía sugerir lo contrario.

Pero un segundo punto, relacionado con el anterior, es que las razones que deben considerarse al determinar si un enunciado de aplicabilidad es correcto incluyen las posibles consecuencias que se derivarían de ello. Porque si hay una determinada decisión $\varphi$ que un tribunal tendría motivos para dictar si la disposición se aplica, con una determinada consecuencia, a un determinado objeto $x$, entonces el hecho de que esa decisión sería, digamos, extremadamente injusta es en sí mismo una consideración que debe tenerse en cuenta al decidir la propia cuestión de la aplicabilidad. Y no considero que este punto sea especialmente controvertido, ya que se reconoce habitualmente que el pensamiento consecuencialista desempeña un papel en lo que comúnmente se denomina "interpretación jurídica". ${ }^{4}$

Dicho esto, sin embargo, una vez que un juez ha establecido que una disposición se aplica, con una consecuencia particular, a un objeto particular, eso en sí mismo dará al tribunal una razón para decidir el caso en cuestión de una manera determinada, y dicha razón debe ser considerada frente a cualquier otra razón que tenga que ver con esa cuestión. $\mathrm{O}$, dicho de otro modo: mientras que los dos puntos anteriores se refieren a las razones (tanto a favor como en contra) que influyen en la aplicación de una determinada disposición, este último punto se refiere a las razones (tanto a favor como en contra) que influyen en la decisión que el tribunal debe adoptar, dado que se aplica una disposición particular. 


\section{Respuesta a José Juan Moreso}

También José Juan Moreso dedica parte de su comentario a mi análisis del deductivismo de reglas en "On the Legal Syllogism". Moreso promete mostrar que "un defensor de la concepción tradicional [...] puede resistir las críticas" que presento contra la visión deductivista de reglas $-\mathrm{y}$, de hecho, que la visión tradicional puede incorporar mis ideas "manteniendo su integridad" (Moreso, 2021, sección 1)—.

Para mostrar este punto, recurre al caso Noara. ${ }^{15}$ Este caso es análogo, dice, al caso Luffe, decisión que analizo en "On the Legal Syllogism" y que, de acuerdo con lo que digo, no puede representarse con el modelo deductivista de reglas. La opinión de Moreso (2021, sección 2, n. 7) es que este tipo de decisiones sí pueden representarse adecuadamente con el modelo del silogismo jurídico, siempre que nos demos cuenta de que el juez considera (o en todo caso debería considerar, si la decisión ha de estar bien justificada sobre la base del derecho existente) "todas las normas aplicables a una determinada situación” (Moreso, 2021, sección 2).

A continuación, Moreso considera, y plantea dudas, acerca de mi afirmación, en “¿Qué es aplicar derecho?”, de que la justificación de los enunciados de aplicabilidad no se basa en enunciados universales de normas, y centra esta discusión en torno a otra decisión real: el caso inglés Jaffa Cakes. $^{16}$

Consideraré cada punto por separado.

\subsection{El caso Noara y el modelo del silogismo judicial}

Aunque la decisión judicial en el caso Noara está escasamente motivada, Moreso dice que "la jueza parece suponer que autorizando el trasplante en este caso está aplicando el art. 4 de la Ley 30/1979” —disposición que

${ }^{15}$ Auto 785/2007 de dieciocho de octubre, Juzgado de Primera Instancia número 17 de Sevilla.

16 United Biscuits (UK) Ltd (No 2) $v$ the Comissioners of Customs and Excise [1991] (LON/91/160) VAT Decision 6344. 
especifica el requisito de que "el donante sea mayor de edad" - "a pesar de que Rocío, la donante, es menor de edad” (Moreso, 2021, sección 2).

No estoy seguro de estar de acuerdo con esta afirmación. Sin embargo, en aras de la discusión, procederé como si la jueza efectivamente considerara que está aplicando esta disposición, y explicaré mi desacuerdo solo al final de esta sección, una vez que haya abordado los argumentos de Moreso.

¿Cómo reconstruiría entonces un deductivista de reglas el argumento en este caso? Moreso dice que yo probablemente sostendría que el deductivista de reglas tendría que reconstruir el argumento como sigue, lo cual, como él señala, es lógicamente falaz:

(1) Todas las personas mayores de edad, en posesión de sus facultades mentales, debidamente informadas y prestando su consentimiento de manera libre y consciente pueden donar órganos para su injerto o implantación en otra persona.

(2) Rocío es una menor de edad, en posesión de sus facultades mentales, debidamente informada y prestando su consentimiento de manera libre y consciente, dispuesta a donar parte de su hígado para ser trasplantado a su hija Noara.

Por lo tanto, de (1) y (2),

(3) Rocío está autorizada a donar parte de su hígado a su hija Noara.

(Moreso, 2021, sección 2).

Y Moreso parece estar de acuerdo conmigo - arguendo, al menosen dos puntos. Primero, los deductivistas de reglas no podrían proponer que la primera premisa se revisara para "excluir la mayoría de edad de la premisa (1)" (Moreso, 2021, sección 2). Y, en segundo lugar, tampoco podrían proponer que la premisa (2) se reescribiera para decir que Rocío es mayor de edad. Cualquiera de las dos estrategias nos daría un argumento lógicamente válido; pero con la primera, la premisa (1) dejaría de ser plausible como enunciado de la regla que supuestamente expresa la disposición correspondiente, y bajo la segunda estrategia la premisa (2) sería simplemente falsa como cuestión de hecho (Moreso, 2021, sección 2).

Pero Moreso, si bien no impugna directamente esta línea de razonamiento contra el deductivismo de reglas, piensa que en el caso Noara "el 
razonamiento de la jueza es insuficiente para mostrar lo que desea mostrar", y que, si la jueza hubiera "mostrado que en el derecho español también existe la causa de justificación del estado necesidad" (Moreso, 2021, sección 2), habría podido ofrecer un argumento que podría ser "reconstruido deductivamente" como un silogismo jurídico.

He aquí cómo Moreso (2021, sección 2) propone reconstruir dicho argumento:

(1") Todas las personas mayores de edad, en posesión de sus facultades mentales, debidamente informadas y prestando su consentimiento de manera libre y consciente pueden donar órganos para su injerto o implantación en otra persona.

(2") Todas las personas están autorizadas a infringir un deber cuando están en estado de necesidad.

(3") Rocío es una menor de edad, en posesión de sus facultades mentales, debidamente informada y prestando su consentimiento de manera libre y consciente, dispuesta a donar parte de su hígado para ser trasplantado a su hija Noara.

(4") Roció está en estado de necesidad, puesto que si no dona parte de su hígado a Noara, entonces Noara morirá.

Por lo tanto, de (1"), (2", (3" y (4"),

(5”) Rocío está autorizada a donar parte de su hígado a su hija Noara.

No veo, sin embargo, que el análisis de Moreso sobre el caso Noara socave en modo alguno mis críticas al deductivismo de reglas y al modelo del silogismo jurídico.

Permítanme comenzar con un par de pequeños puntos respecto de la reconstrucción ofrecida por Moreso de lo que él consideraría el argumento correcto, en el derecho español, que el juez debería haber utilizado para decidir el caso Noara.

La causa de justificación de estado de necesidad está pensada para hacer jurídicamente permisible una acción que, no obstante, realiza un tipo penal. Pero mientras que esto probablemente sería cierto tanto para el médico que llevaría a cabo la operación de trasplante (y digo "probablemente" porque 
carezco de los conocimientos pertinentes del derecho español), no sería, sospecho, cierto para la propia acción de Rocío de donar parte de su hígado a su hija. Es más probable que se considerase a Rocío como víctima y no como agente del delito de tráfico de órganos. Pero el caso Noara se refería explícitamente a la cuestión de si la propia Rocío estaba autorizada a donar parte de su hígado a su hija. Así lo dice claramente la jueza en la parte dispositiva de su sentencia: "Debo autorizar y autorizo a Gabriela la donación de hígado solicitada para su hija Verónica, quedando así suplida su falta de capacidad, integrándose el consentimiento de la misma”. Es también lo que Moreso toma como conclusión de su propia reconstrucción. Parece extraño, por tanto, sugerir que el caso debería haberse decidido apelando a la figura del estado de necesidad. ${ }^{17}$

Téngase en cuenta, además, que la jueza del caso Noara claramente considera que su decisión de hacer permisible que Rocío done parte de su hígado a su hija significa que la donación está legalmente autorizada desde el inicio. Y aunque, como dice Moreso, apelar a la figura del estado de necesidad, que es una causa de justificación, significaría también que la acción correspondiente estaría permitida, hay una diferencia jurídicamente relevante (y comúnmente aceptada) entre (a) las acciones que están permitidas porque, aunque incumplen un deber, se encuadran en una causa de justificación legalmente reconocida, y (b) las acciones que están jurídicamente permitidas porque, de entrada, no incumplen ningún deber. ${ }^{18}$

17 Por no mencionar que tampoco me parece evidente que la interpretación que hace Moreso del artículo cuarto a) de la Ley 30/1979 en el sentido de que impone "un deber" (como él dice) "que impide a los menores de edad ser donantes" sea necesariamente acertada. Yo consideraría más naturalmente que impone una incapacidad, es decir, la ausencia de una facultad para consentir válidamente y, por tanto, hacer permisible la obtención de órganos. (Y, por cierto, la reconstrucción propuesta por Moreso no es, tal como se la ha presentado, un argumento formalmente válido. Estoy de acuerdo, sin embargo, en que las premisas podrían reescribirse fácilmente para hacerlo formalmente válido. Pero eso supondría, entre otras cosas, reformular (1) como un enunciado acerca del deber de no donar, lo que haría más destacable el punto que acabo de exponer sobre la plausibilidad de entender el artículo cuarto a) de la Ley 30/1979 de esa manera).

${ }^{18}$ Que la distinción es jurídica y dogmáticamente relevante es el punto principal de la famosa comparación de Welzel entre matar a un mosquito, por un lado, y matar a una persona en defensa propia: véase Welzel (1955, p. 211) y Welzel (1969, p. 81). 
Así que no considero que con su reconstrucción Moreso esté simplemente ofreciendo un argumento diferente (y supuestamente mejor) para la misma conclusión a la que llegó la jueza en el caso Noara. Moreso escribe que la solución de la jueza "es correcta, conforme al derecho español, pero la jueza debería haber razonado de otro modo" (Moreso 2021, sección 2). Pero si, como parece, la conclusión del argumento que propone es que la acción de Rocío debe seguir siendo considerada como un incumplimiento (justificado) de un deber, y si, como también parece, la conclusión de la jueza fue que la acción de Rocío no constituía un incumplimiento de ningún deber para empezar - entonces son argumentos para conclusiones diferentes-.

Pero estos, como he dicho, son puntos menores sobre el tratamiento propuesto por el propio Moreso para el caso. Porque incluso si concedemos a Moreso todos sus puntos sustanciales, no ha socavado mi crítica al deductivismo de reglas.

¿Por qué no? Consideremos de nuevo lo que Moreso dice que yo sugeriría como la reconstrucción que los deductivistas de reglas ofrecerían del argumento de la jueza en el caso Noara:

(1) Todas las personas mayores de edad, en posesión de sus facultades mentales, debidamente informadas y prestando su consentimiento de manera libre y consciente pueden donar órganos para su injerto o implantación en otra persona.

(2) Rocío es una menor de edad, en posesión de sus facultades mentales, debidamente informada y prestando su consentimiento de manera libre y consciente, dispuesta a donar parte de su hígado para ser trasplantado a su hija Noara.

Por lo tanto, de (1) y (2),

(3) Rocío está autorizada a donar parte de su hígado a su hija Noara.

Esto es, como dice Moreso, un argumento lógicamente inválido; pero no es ese el problema básico del deductivismo de reglas. El problema básico con el deductivismo de reglas, como explico en "On the Legal Syllogism" y también he mencionado ya en varios puntos del presente artículo, es que atribuye a los jueces un compromiso con enunciados de reglas universales. 
Y la reconstrucción que ofrece Moreso hace precisamente lo mismo. Atribuye a la jueza en el caso Noara un compromiso con al menos dos de esos enunciados: la premisa (1"), y la premisa (2"). Pero no veo que tengamos ni buenas razones ni buenos fundamentos para considerar que la jueza está comprometida con la verdad de tales enunciados universales, por las razones que ya he repasado ampliamente (especialmente en la sección 2.2) al discutir algunas de las observaciones críticas de Hugo Zuleta.

Y, en términos más generales, digo claramente en "On the Legal Syllogism" que mi recurso a ejemplos como el del caso Luffe tiene por objeto simplemente mostrar con mayor claridad algo que también está presente, aunque no de manera manifiesta, en la reconstrucción de casos más sencillos por parte de los deductivistas de reglas. Esto también es un punto que ya he señalado anteriormente (al final de la sección 2.7). Para citar de nuevo el pasaje pertinente de "On the Legal Syllogism", el modelo del silogismo falla incluso cuando los deductivistas de reglas no tienen ninguna "duda de que la regla relevante se aplica al caso en cuestión". "Esos", escribí, "son casos que el modelo solo parece captar bien; la apariencia es engañosa" (Duarte d'Almeida, 2019, p. 351). Y eso es cierto también de la reconstrucción propuesta por Moreso.

Mencioné al principio de esta sección que no estoy seguro de que Moreso tenga razón en que la jueza del caso Noara supusiera que estaba “aplicando el art. 4 de la Ley 30/1979” (Moreso, 2021, sección 2). En mi opinión, estaba haciendo algo diferente. Simplemente estaba dando la autorización que la propia Rocío no podía, debido a su edad, válidamente proporcionar. Esto se corresponde con lo que la jueza dice explícitamente que está haciendo: el efecto de su decisión es que "queda [] así suplida [la] falta de capacidad" de Rocío.

Pero si esto es así, la jueza no está aplicando el artículo 4 de la Ley 30/1979. Más bien, con su decisión, está haciendo que el artículo sea aplicable a este trasplante concreto, con la consecuencia de que pasa ahora a estar jurídicamente permitido (que es precisamente el mismo efecto que habría tenido el consentimiento de la propia Rocío, de haber sido mayor de edad). Esto también explicaría la escasez de argumentos reales en la decisión. Y significaría, por supuesto, que el caso Noara no es relevantemente análogo a casos como Luffe después de todo. 


\subsection{El caso Jaffa Cakes y la justificación de los enunciados de aplicabilidad}

El segundo punto de Moreso se refiere a la justificación de lo que yo llamo "enunciados de aplicabilidad". Introduce el caso Jaffa Cakes, ofrece una reconstrucción del argumento del tribunal según el modelo que propongo, y dice que "no t [iene] mayores objeciones a esta reconstrucción" (Moreso, 2021, sección 3) ${ }^{19}$. Tomo su acuerdo (que es bienvenido) como reflejo e ilustración del hecho de que, como expliqué anteriormente en la sección 3.2 , los deductivistas de reglas no tienen ninguna razón para rechazar la visión que propongo de la estructura de la justificación de las decisiones que aplican derecho.

Sin embargo, la cuestión importante que hay que plantear, dice Moreso, es:

¿[C]ómo se justifica (2), la premisa que dice que [la Schedule n. 5 de la Value Added Tax Act 1983] es aplicable a[1] caso [Jaffa Cakes]? Y, ¿por qué Luís piensa que no se justifica acudiendo a pautas generales? (Moreso, 2021, sección 3).

19 Una objeción menor que hace Moreso es que mi inclusión de la cláusula "no hay consideraciones compensatorias" en las premisas (4) y (5) parece indicar un respaldo a una estrategia que yo mismo he refutado en trabajos anteriores, y en particular en mi libro Allowing for Exceptions: A Theory of Defences and Defeasibility in Law (Duarte d'Almeida, 2015a). Es un comentario justo, pero me temo que falla en el blanco. En primer lugar, mi rechazo, en Allowing for Exceptions a estrategias como la de Richard Holton (que sugiere que la inclusión, en un argumento reconstruido, de una cláusula "That's it" nos permite ver que el efecto "suplantador" de las excepciones es compatible con la justificación deductiva) se refería al tema de las excepciones y la derrotabilidad, no a la ponderación de razones aplicables. Si se tiene una razón para practicar una acción $\varphi$ y una razón de mayor peso (y, por tanto, compensatoria) para no realizar esa acción $\varphi$, entonces, todo considerado, no se debe realizar la acción $\varphi$; pero esto no hace que la razón de mayor fuerza en sentido contrario sea una "excepción", en el sentido relevante de la palabra. Y, en segundo lugar, no tengo nada, en principio, en contra de las cláusulas ceteris paribus (de las que la propuesta por Holton es un ejemplo), y de hecho en Allowing for Exceptions yo mismo introduzco la noción de cláusula ceteris ignotis, en la que me baso como parte de mi explicación de la derrotabilidad en el derecho. Véase Duarte d'Almeida (2015a, cap. 6) (y la sección 6.3. en particular para una discusión de las cláusulas “That’s it”), así como Holton, 2002. Discuto la propuesta de Holton con más detalle que en mi libro en mi artículo "Exceptions and Supersession" (Duarte d'Almeida, 2014), que está también disponible en traducción al español (Duarte d'Almeida, 2015b). 
Y en contra de mi opinión de que los tribunales no justifican tales enunciados de aplicabilidad sobre la base de premisas universales, Moreso dice - coincidiendo con un punto de Joseph Raz- que para que la premisa "que dice que la pauta contenida en la Schedule n. 5 de la Value Added Tax Act 1983 es aplicable" a los Jaffa Cakes "sea inteligible, es necesario que se apoye en algunas razones que son de carácter general" (Moreso, 2021, sección 3).

Pero no estoy en desacuerdo con esto. No sostengo que la justificación de los enunciados de aplicabilidad no se apoye en razones de carácter general; de hecho, creo que debe hacerlo. Lo que sí sostengo es que, aunque los tribunales recurren apropiadamente a tales razones, no ofrecen formulaciones de enunciados universales que especifiquen todas y solo las características relevantes del caso en cuestión. De hecho, esto es precisamente lo que vemos en el caso Jaffa Cakes. Consideremos cómo el propio Moreso describe la línea de argumentación del tribunal:

El Tribunal toma en consideración algunas semejanzas de los Jaffa Cakes con las galletas, como que son de tamaño más semejante a las galletas, se comen con los dedos como las galletas, se empaquetan como ellas y parecen en los comercios que las venden agrupados junto con las galletas y no con los pasteles. Hay, sin embargo, otros elementos que las asemejan a los pasteles, cuando pasa el tiempo al igual que los pasteles se ponen duras y resecas, mientras las galletas con el paso del tiempo se ablandan (Moreso, 2021, sección 3).

Este es, en mi opinión, un ejemplo típico de cómo los tribunales justifican los enunciados de aplicabilidad. Tienen "en cuenta" diferentes "hechos y consideraciones" - para usar el lenguaje que el tribunal del caso Jaffa Cakes emplea explícitamente- que estiman relevantes para resolver el asunto en cuestión. Las consideraciones que menciona el tribunal son razones de aplicación general. El hecho de que una pieza de confitería cubierta de chocolate tenga un tamaño similar al de las galletas, que se coma con los dedos y que se exponga normalmente para su venta junto con galletas, son razones, dice el tribunal, para no aplicarles la pauta contenida en la Schedule 5, mientras que el hecho de que se endurezcan en lugar de ablandarse con el tiempo es una razón para aplicar esa disposición. Pero 
aun así, el tribunal no se aventura a formular un enunciado universal del tipo: "siempre que una pieza de confitería que es más similar en tamaño a una galleta que a un bizcocho, se come con los dedos, y se expone para su venta junto con galletas, se le aplica la Schedule 5", ya que bien puede ser posible que surja otro caso en el que estén presentes todos esos puntos de similitud y disimilitud, pero que también presente algunos otros puntos de similitud relevantes que justificarían una conclusión diferente. El tribunal tampoco se compromete con un enunciado universal del tipo "siempre que un producto de confitería que se asemeja más a una galleta que a un bizcocho, se come con los dedos y se expone para su venta junto con galletas, entonces, ceteris paribus, se le aplica la Schedule 5", ya que no puede afirmarse con certeza que esas mismas características tengan necesariamente la misma fuerza (y dirección) al proveer de razones en otro caso particular que pueda surgir en el futuro. Y ni siquiera se puede considerar que el tribunal se comprometa con un enunciado universal aún más general del tipo "Siempre que una pieza de confitería se parezca más a un pastel que a una galleta, se le aplica el Schedule 5", ya que la similitud relevante puede ser solo uno de los posibles criterios pertinentes que se aplicarían adecuadamente en algún otro caso.

Pero es cierto, lo sé, que en mis artículos no he aportado nada que ni siquiera empiece a proporcionar una explicación clara del razonamiento y los argumentos que se utilizarían para justificar los enunciados de aplicabilidad. Esa también es una tarea para futuros trabajos.

\section{Bibliografía}

Carnap, R. (1962). Logical Foundations of Probability. 2 ed. Chicago, Toronto: The University of Chicago Press; Routledge and Keegan Paul. Carroll, L. (1895). What the Tortoise Said to Achilles. Mind, 4(14), 278-280. Duarte d'Almeida, L. (2014). Exceptions and Supersession. Ragion Pratica, 42, 187-203.

Duarte d'Almeida, L. (2015a). Allowing for Exceptions: A Theory of Defences and Defeasibility in Law. Oxford: Oxford University Press. 
Duarte d'Almeida, L. (2015b). Excepciones y superación. En P. Luque (ed), Particularismo: ensayos de filosofía del derecho y filosofía moral (pp. 133-150). Barcelona: Marcial Pons.

Duarte d'Almeida, L. (2019). On the Legal Syllogism. En D. Plunkett, S. Shapiro y K. Toh (eds), Dimensions of Normativity: New Essays on Metaethics and Jurisprudence (pp. 335-364). Nueva York: Oxford University Press.

Hart, H. L. A. (1955). Theory and Definition in Jurisprudence. Proceedings of the Aristotelian Society: Supplementary Volumes, 29, 239-264.

Hart, H. L. A. (1967). Problems of the Philosophy of Law. Republicado en H. L. A. Hart. (1983) Essays in Jurisprudence and Philosophy. Oxford: Clarendon Press.

Hart, H. L. A. (2012 [1961]). The Concept of Law. 3 ed. Oxford: Oxford University Press.

Hempel, C. G. (1979 [1965]). La explicación científica. Barcelona: Paidós.

Holton, R. (2002). Principles and Particularisms. Proceedings of the Aristotelian Society: Supplementary Volumes, 67, 191-209.

MacCormick, N. (2005). Rhetoric and the Rule of Law: A Theory of Legal Reasoning. Oxford: Oxford University Press.

Moreso, J. J. (2021). Aplicación del derecho y los casos Noara y Jaffa Cakes. Discusiones, 27(2).

Scataglini, G. (2021). Aquiles, la Tortuga y la aplicación de normas jurídicas como un 'asunto particularista' sin particularismo. Discusiones, 27(2).

Simpson, A. W. B. (1961). The Ratio Decidendi of a Case and the Doctrine of Binding Precedent. En A. C. Guest (ed.), Oxford Essays in Jurisprudence (pp. 148-175). Oxford: Oxford University Press.

Theobald, W. (1836). A Practical Treatise on the Poor Laws, as Altered by the Poor Law Amendment Act, and Other Recent Statutes. London: Stevens \& Sons.

Welzel, H. (1955). Die Regelung von Vorsatz und Irrtum im Strafrecht als legislatorisches Problem. Zeitschrift für die gesamte Strafrechtswissenschaft, 76, 196-228.

Welzel, H. (1969). Das Deutsche Strafrecht: Eine Systematische Darstellung, 11th edition. Berlín: Walter de Gruyter. 
Aplicación del derecho y la justificación de las decisiones judiciales

Williams, G. (1988). The Logic of "Exceptions". Cambridge Law Journal, $47,261-95$

Zuleta, H. (2021). Comentario a ¿Que es aplicar derecho?’ de Luís Duarte d'Almeida. Discusiones, 27(2). 